\title{
BLOCKCHAIN VS ISO 9001:2015 BLOCKCHAIN VS ISO 9001:2015
}

\author{
Víctor Gisbert Soler \\ Doctor Ingeniero Industrial. \\ Profesor Universitat Politècnica de València. \\ E-mail: vgisber@eio.upv.es
}

Ana Isabel Pérez Molina Doctora en Ingeniería en Organización Industrial. Profesora Universitat Politècnica de València.

E-mail: anpemo@eio.upv.es

\section{Gitación sugerida:}

Gisbert Soler, V. y Pérez Molina, A. I. (2019). Blockchain vs ISO 9001:2015. 3C Tecnología. Glosas de innovación aplicadas a la pyme, 8(2), pp. 36-49. doi: http://dx.doi.org/10.17993/3ctecno/2019.v8n2e30.36-49 


\section{RESUMEN}

En la presente comunicación se realiza una pormenorizada introducción sobre la tecnología Blockchain, los beneficios de su utilización, así como su utilización en los llamados contratos inteligentes.

Se desarrolla de forma detallada las posibles aplicaciones de Blockchain ligadas a un sistema de gestión de la calidad basado en la norma ISO 9001:2015.

Se realiza un estudio sobre las principales características principales del uso, funciones clave en los términos de fabricación en general, y en gestión de la calidad, para concluir sobre diferentes casos de uso de fabricación avanzada y gestión de aprovisionamientos.

\section{PALABRAS CLAVE}

Blockchain, ISO 9001, Contratos inteligentes.

\section{ABSTRACT}

In the present communication a detailed introduction is made about the Blockchain technology, the benefits of its use, as well as its use in the so-called intelligent contracts.

The possible Blockchain applications linked to a quality management system based on ISO 9001: 2015 are developed in detail.

A study is made on the main characteristics of the use, key functions in the terms of manufacturing in general, and in quality management, to conclude on different cases of use of advanced manufacturing and procurement management.

\section{KEYWORDS}

Blockchain, ISO 9001, Intelligent contracts. 


\section{INTRODUCCIÓN}

Blockchain se asocia comúnmente con la transferencia de activos digitales. Se trata esencialmente de bloques de código de programación que están vinculados entre sí de manera segura.

De esta forma, un blockchain puede entregar un registro inmutable e irreversible para activos digitales y puede tener innumerables aplicaciones prácticas tanto en fabricación como en aseguramiento y control de calidad.

En este artículo se analizan las aspiraciones digitales en aseguramiento y control de calidad y como podría mejorarse mediante la aplicación de blockchains.

El principal hallazgo y conclusiones es que blockchain se puede utilizar para proporcionar herramientas avanzadas para la gestión de la calidad, con verificación de propiedad segura, piezas, validación de pedidos, inspecciones realizadas, controles de proceso, etc.

Podemos concluir que las aplicaciones de blockchain pueden ofrecer un método robusto y resistente de la indexación del historial de registros que se puede distribuir y almacenar en Internet. Esta capacidad de indexación lo hace especialmente útil en gestión de la calidad, como posteriormente veremos.

El blockchain ayuda en la identificación y por lo tanto la eliminación de la falsificación. También como resultado de la digitalización y propiedades de los registros, blockchain tiene muchas otras aplicaciones potenciales, ya que su uso agrega rigor a la medición y trazabilidad que se requiere en la fabricación. También puede facilitar la personalización exigida en cualquier fabricación, pudiéndose utilizar para rastrear tanto el "uso" como el "efecto" en la cadena de suministro.

Blockchain tiene muchas aplicaciones potenciales, ya que su uso agrega rigor a la medición y trazabilidad que se requiere en la fabricación.

$\mathrm{Al}$ igual que en las aplicaciones financieras, los fabricantes pueden utilizar blockchain para ver el estado de los pagos, facturas, documentos y datos digitalizados, se puede utilizar para dar una visibilidad detallada del producto a través de toda la cadena de suministro, la cual se ve reforzada por capacidad de blockchain para almacenar y procesar todos los registros y datos inherentes a esa producción. 
$\mathrm{Al}$ no poder ninguna parte modificar o eliminar ningún registro sin consenso, el nivel de transparencia proporcionado por blockchain, además de facilitar la trazabilidad, puede ayudar a reducir el fraude y los errores.

También podría ayudar a reducir el tiempo en los productos están tanto en producción como en almacenamiento y distribución, al mejorar la gestión de inventario y reduciendo tiempos muertos y desperdicios.

Este documento propone casos avanzados de uso de fabricación y gestión de la calidad para ilustrar el potencial de Blockchain.

En primer lugar, Blockchain nos puede servir para ver el estado de los bienes a través de la cadena de valor. Esto a su vez arroja visibilidad sobre donde los componentes están en el proceso de valor agregado.

En segundo lugar, Blockchain puede ayudarnos para el análisis de series de tiempo. En gestión de la calidad, las series de tiempo se utilizan en el control del producto y prestación del servicio.

Las variables monitoreadas son activos digitales, que incluyen, por ejemplo, cualquier variable que quiera ser controlada, como unos diámetros de componentes de precisión, o atributos, características de calidad que no pueden ser revisadas mediante una escala de medida, tanto en materia prima, producto en proceso y terminado.

Los gráficos de control incluyen series de tiempo con límites superiores e inferiores de control, que como hemos dicho pueden ser variables de proceso u otro tipo de indicadores de eficiencia de proceso, kpi's, como tiempo que lleva procesar un pedido, cambio rápido de formato, materia prima utilizada, resultado de una inspección por muestreo, etc.

\section{LOS BENEFICIOS DE USAR BLOCKCHAIN}

Blockchain es una tecnología habilitadora que puede ayudar a reducir la carga manual del cumplimiento y procesos de control e inspección (2 Deloitte).

Es válido donde quiera que haya relaciones basadas en la confianza en el suministro, que deba ser transparente o verificado por un tercero mientras se mantiene confidencialidad y / o intermediación. 
Un ejemplo es una empresa que busca obtener un servicio de logística donde el envío, por ejemplo, de alimentos congelados, la temperatura nunca deba ser superior a cero centígrados. Esto puede ser verificado automáticamente a través de Ia tecnología Blockchain.

Así pues, Blockchain puede ayudar a la selección de proveedores en base a hechos constatables, en lugar del habitual procedimiento de evaluación de proveedores basado en garantías o auditorías de cumplimiento.

La potencialidad de Blockchain permitirá mejorar el valor de los productos adquiridos a subcontratistas al existir plena transparencia en su realización.

\section{CONTRATOS INTELIGENTES}

Se ha prestado considerable atención al potencial de la tecnología blockchain para ser utilizada en contratos inteligentes. Un contrato inteligente es esencialmente un acuerdo escrito en código de programación y entregado por un blockchain (2 Ethereum).

$\mathrm{Al}$ igual que el blockchain financiero, en el que una parte tiene posesión de un artículo y puede privar a otro de parte de acceso a la propiedad, se puede proceder de igual manera en gestión de la calidad.

En estos casos, el contrato inteligente sirve como una prueba de acuerdo firmado criptográficamente entre las dos partes. Desde una perspectiva tecnológica, los contratos inteligentes a menudo se ven como fuera de la jurisdicción convencional, pero desde una perspectiva legal, existe el derecho de acceso a la justicia ordinaria.

El proceso de permitir que un tribunal se pronuncie sobre un contrato inteligente, donde los términos no pueden ser alterados presenta un desafio significativo para futuras investigaciones.

Desde una perspectiva tecnológica, los contratos inteligentes a menudo se ven como fuera de la jurisdicción convencional, pero desde una perspectiva legal, existe el derecho de acceso a la justicia ordinaria. 


\section{APLICACIONES DE GESTIÓN DE LA CALIDAD EXISTENTES PARA BLOCKCHAIN}

En esta sección exploramos los usos de blockchain que ya se han implementado en la gestión de la calidad (3 Cointelegraph).

Algunos ya están disponibles en el mercado en este momento, otros se han propuesto, pero aún no se han implementado.

\section{Procedencia del producto al por menor}

Hay una serie de productos y servicios en el mercado de hoy cuyo valor está ligado, en mayor o menor grado, a su 'procedencia', como por ejemplo productos alimenticios producidos de manera sostenible, artículos de lujo, etc.

La tecnología Blockchain se puede usar para establecer la procedencia de productos manufacturados avanzados. En la actualidad hay varias empresas que ya operan en el contexto de la tecnología blockchain para determinar la procedencia.

Dichas empresas se centran en la responsabilidad de la cadena de suministro de los productos, trabajan con proveedores para recopilar información del proceso de la cadena de suministro y compartir esta información con los clientes en el punto de venta del producto.

Usando este enfoque, blockchain se puede usar para rastrear materiales y productos, y funciona a nivel de artículo. Proporciona una experiencia orientada al cliente, que detalla los pasos clave en la producción de un producto.

La cadena de suministro se modela como compuesta por productores, fabricantes, registradores, organizaciones de estándares, certificadores y auditores, y clientes.

Los registradores actúan como gestores de confianza para verificar la identidad y las credenciales de los otros participantes nombrados. Las organizaciones de estándares definen requisitos para una aprobación dada (por ejemplo, no pruebas con animales en productos farmacéuticos), y las organizaciones permiten que se agreguen lotes de productos a este grupo. El proceso de aprobación de un fabricante para un 
estándar dado puede requerir una certificación o auditoría, que se llevaría a cabo por un auditor. Una verificación exitosa da como resultado un fabricante que está registrado en una entidad de certificación.

Después de la certificación, un productor tiene un programa de producción aprobado, que está certificado para una capacidad de producción determinada durante un período de tiempo.

\section{$\underline{\text { Transparencia en la cadena de suministro }}$}

Cada vez más los fabricantes quieren que la procedencia de los productos esté más disponible para el usuario final.

Como se señaló en la introducción, la gestión de la calidad proporciona una mayor cantidad de valor para el cliente a través de sus actividades. Esto se logra mediante el despliegue de altos niveles de tecnología, habilidad y conocimiento.

En una cadena de suministro tradicional, esto es aceptado por el cliente basado en la confianza de las distintas certificaciones, que han llevado a algunos escándalos en los últimos años por la no validez o manipulación de esas certificaciones.

El uso de blockchain proporciona una manera de abordar esta brecha entre confianza percibida y comportamiento real. Por ejemplo, un índice de las partes involucradas en la producción de un producto se puede registrar de forma indeleble en blockchain del producto.

Esta capacidad puede extenderse para proporcionar alguna evidencia que respalde cualquier reclamación hecha sobre productos o servicios previstos.

\section{$\underline{\text { Historial de servicio rastreable }}$}

El uso de registros de la actividad de servicio habilitados por blockchain en un activo que ya ha sido identificado como posible uso por parte de empresas fabricantes. En la larga vida de un vehículo comercial, el historial de servicio y las piezas usadas se convierten en un patrón complejo de uso o actividad, mantenimiento y reemplazo de piezas. Con las tecnologías actuales esto requiere disciplina para mantener y no es difícil de falsificar. 
Las soluciones Blockchain ayudan siendo esencialmente automatizadas. Es cierto que las partes interesadas todavía tienen para acordar el uso de la cadena de bloques, pero mantiene un índice inalterable de eventos en el que la secuencia se mantiene. Esto apoyará una evaluación sostenida en información veraz de los valores de los activos a lo largo de su vida.

Fabricación habilitada para IoT

Internet de las cosas (IoT) es una tendencia tecnológica en desarrollo que ha sido ampliamente predicha y aún no se ha implementado.

La aplicación de IoT dentro de la fabricación es un paso natural y hoy existen varios ejemplos de aplicaciones basadas la interacción con la maquinaria de producción. Un ejemplo típico es una máquina CNC de mensajes de texto al operario cuando se requiere soporte para un próximo cambio de herramientas. Se puede considerar parte de la próxima generación de evolución de la industria (industria 4.0).

La aplicación de loT dentro de la fabricación se puede considerar parte de la próxima generación de evolución de la industria (industria 4.0).

Blockchain ha sido identificada como tecnología de apoyo, proponiéndose una plataforma de uso general para el uso de blockchain en el IoT industrial que permite la fabricación "bajo demanda".

Esto ha sido descrito de diversas maneras como fabricación en nube y fabricación distribuida, y se considera que ofrece importantes flexibilidad y ventajas de costo sobre la fabricación típica.

\section{CARACTERÍSTICAS PRINCIPALES DEL USO}

Funciones clave en los términos de fabricación en general, y en gestión de la calidad en particular, son 5 (Ferrer, 2018):

- Resistencia a la manipulación. La naturaleza distribuida de la base de datos hace que sea muy difícil cambiar registros de transacciones pasadas. 
- Trazabilidad incorporada. Un corolario de la resistencia a la manipulación es que la trazabilidad de las transacciones puede ser incorporada a la cadena de bloques. Cada transacción se registra y no se puede modificar en la práctica, existiendo un rastro de tales transacciones.

- Transparencia en lugar de confianza. Sobre la base de las dos características anteriores, la cadena de bloques puede ser visto como una historia transparente de lo que ha sucedido. Esta transparencia puede ser lograda a través del uso de claves criptográficas.

\section{CASOS DE USO DE FABRICACIÓN AVANZADA Y GESTIÓN DE APROVISIONAMIENTOS}

\section{Economía circular / refabricación}

El término "economía circular" implica el movimiento desde la extracción de las materias primas, su posterior conformado para convertirla en productos que luego son utilizados y posteriormente eliminados una vez se cierra el ciclo.

La refabricación ofrece el potencial de reducir en gran medida la utilización de recursos, al tiempo que proporciona la función y garantías de nuevos productos. Es una industria en claro crecimiento como son los casos de los sectores del automóvil, electrónica, sector aeroespacial, etc.

Dentro de la refabricación nos podemos encontrar:

- Remanufactura: devuelve al producto usado una nueva especificación de rendimiento y le da al producto resultante una garantía que es al menos igual a la de un producto nuevo equivalente.

- El reacondicionamiento: devuelve un producto a unas condiciones de funcionamiento satisfactorias que pueden ser inferiores a la especificación original y a da una garantía menor que la de nueva fabricación de producto.

- La reparación corrige las fallas especificadas en un producto y da una garantía menor al nuevo producto manufacturado que puede no cubrir todo el producto. 
- El reciclaje: recupera materiales para el propósito original o un nuevo propósito.

El factor clave de éxito en cualquier cadena de refabricación es la condición y el estado de los productos devueltos, que aumentará el valor al tener pleno conocimiento y, por tanto confianza, en que los artículos devueltos son correctos, al incorporar:

- Un registro de la especificación del producto devuelto que es inmutable y preciso.

- Más información transparente sobre el estado del producto devuelto.

- Claridad del estado del producto devuelto después de la remanufactura.

\section{$\underline{\text { Servicios de ingeniería de por vida }}$}

Productos complejos, por ejemplo, barcos, ferrocarriles, centrales eléctricas, etc. se caracterizan por ciclos de vida operacionales durante los cuales los sistemas que componen el producto pueden necesitar ser modificados, reparados o reemplazados.

Actualmente la gestión del producto, la configuración y los datos relacionados se realizan a través de registros y auditorías que confirman la trazabilidad y confianza en las operaciones. Estas operaciones suelen ser procesos sofisticados para mantener y administrar, y a menudo necesitan ser restringidos en el alcance para ser prácticos.

Usos actuales propuestos de blockchain podrían mantener la información del producto de una manera que sea independiente, actualizada y veraz.

\section{$\underline{\text { Sistemas de servicio del producto }}$}

Existe una tendencia creciente a ofrecer productos como servicio en lugar de venderlos a los clientes. Estas proposiciones comerciales tienen su propia lógica y no requieren una distribución.

Sin embargo, el uso de blockchains ofrece un cierto potencial de beneficios para los sistemas de servicio del producto, como son la ampliación de los sistemas de servicio de productos a mercados más amplios y la capacidad de desintermediación que elimina la función de corretaje en un mercado. 


\section{CONCLUSIONES}

La principal la conclusión es que blockchain ofrece ventajas significativas para la gestión de la calidad.

Las oportunidades que identificamos provienen de factores de reducción de costos, auditorías, validaciones y controles sobre el producto. Todo eso sin tener en cuenta otras ventajas como son una mayor capacidad de respuesta, valor agregado en el producto final y nuevos servicios basados en la información de blockchain.

Por eso entendemos que Blockchain es perfectamente compatible con la gestión de la calidad con el fin de lograr el máximo beneficio. Entendemos que en las etapas intermedias pueden ser mejoradas y "conectadas" a través de interfaces blockchain en sistemas de gestión de la producción, tipo ERP, a nivel corporativo.

También encontramos un gran beneficio para evitar la falsificación y copia al usar esta tecnología, reduciendo de forma importante el valor de las copias 'clonadas'.

A corto plazo los beneficios se centran en la autentificación de cadena de suministro, mientras que a largo plazo están más relacionados con el ciclo de vida del producto. 


\section{REFERENCIAS BIBLIOGRÁFICAS}

Ferrer, A. (2018). Aplicación del blockchain a los datos. Recuperado de: https://es.slideshare.net/maredata/ antonia-ferrer-aplicacin-del-blockchain-a-los-datos

Rodríguez Roldán, D. (s.f.). Blockchain: cambiando los modelos de relacióny confianza. Recuperado de: https:// www2.deloitte.com/es/es/pages/governance-risk-and-compliance/articles/blockchain-cambiandomodelos-relacion-confianza.html

Rojas, E. (2019). ¿Qué son los contratos inteligentes o «smart contracts?? Guía completa. Recuperado de: https:// es.cointelegraph.com/explained/what-is-a-smart-contract 


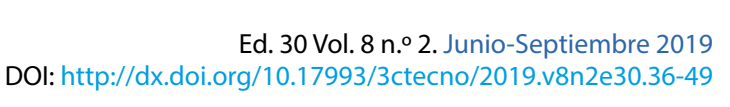

